\title{
Theoretical models of ferromagnetic III-V semiconductors
}

\author{
T. Jungwirth ${ }^{1,2}$, Jairo Sinova ${ }^{2}$, J. Kučera ${ }^{1}$ and A. H. MacDonald ${ }^{2}$ \\ ${ }^{1}$ Institute of Physics ASCR, Cukrovarnická 10, 162 53 Praha 6, Czech Republic \\ ${ }^{2}$ Department of Physics, The University of Texas, Austin, TX 78712
}

(November 12, 2018)

\begin{abstract}
Recent materials research has advanced the maximum ferromagnetic transition temperature in semiconductors containing magnetic elements toward room temperature. Reaching this goal would make information technology applications of these materials likely. In this article we briefly review the status of work over the past five years which has attempted to achieve a theoretical understanding of these complex magnetic systems. The basic microscopic origins of ferromagnetism in the (III,Mn) V compounds that have the highest transition temperatures appear to be well understood, and efficient computation methods have been developed which are able to model their magnetic, transport, and optical properties. However many questions remain.
\end{abstract}

It is hoped that the emerging field of semiconductor spintronics, which studies the controlled flow of charge and spin in a semiconductor, will lead to the development of new non-volatile, high-density, and high-speed information technologies. An important milestone in this field was the discovery five years ago of ferromagnetism in Mn-doped, p-type GaAs observed at temperatures in excess of 100 Kelvin [1]. Ferromagnetism at room temperature with full participation of itinerant carriers would be a major breakthrough in semiconductor spintronics. With this aim, intensive material research is currently in progress on transition metal doped III-V semiconductors. In this brief review we present a snap shot of the theoretical part of this endeavor, which has progressed hand in hand with experimental developments.

A qualitative picture of the electronic structure of III-V diluted magnetic semiconductors (DMSs) was proposed by Dietl and coworkers $[2,3]$. Their model is based on the internal reference rule [6] which states that energy levels derived from the d-shell of the magnetic ion are constant across semiconductor compound families with properly aligned bands. The application of this rule to III- $\mathrm{V}$ materials is illustrated in Fig 1 for ionized magnetic impurities substituted on cation sites [2-5]. The position of the $\mathrm{A}_{2}$ level for $\mathrm{Mn}$ in GaAs, deep in the valence band, suggests that $\mathrm{Mn}$ in GaAs is 2+, that its d-shell is occupied by five electrons, and that incorporation of $\mathrm{Mn}$ in this and many other (III,Mn)V compounds will result a large density of valence band holes that can mediate ferromagnetic coupling between the $S=5 / 2 \mathrm{Mn}$ local moments. For low carrier densities, these valence band holes will be bound to the Mn ion, leading to shallow acceptor levels. This model of carrier-induced ferromagnetism is now fairly well established for $(\mathrm{Ga}, \mathrm{Mn}) \mathrm{As}$ as a result of electron paramagnetic resonance experiments [7], xray magnetic circular dichroism measurements $[8,9]$, and magneto-transport data [10-13].
The acceptor impurity levels of Fe and Co $[2,3,6,4]$ are unlikely to lead to high valence band hole concentrations in arsenides or antimonides, as shown in Fig. 1. The possible coexistence of acceptor and neutral magnetic impurities suggests that a ferromagnetic double-exchange mechanism can dominate in this case [14]. The scenario, however, has not yet been confirmed experimentally. For nitrides and phosphides the ionization energy of the acceptor levels is even higher and the $\mathrm{A}_{2} \mathrm{Mn}$ impurity level is not deep in the valence band, making it likely that d-charge fluctuations cannot be neglected. Experimentally, ferromagnetism with critical temperatures exceeding room-temperature has been claimed in $(\mathrm{Ga}, \mathrm{Mn}) \mathrm{N}$ $[15,16]$ on the basis of hysteresis in magnetization measurements, however, the electronic configuration of $\mathrm{Mn}$ impurity in this compound has not yet been conclusively established, and these claims have not yet been widely confirmed. The observation of the $p$-type conduction achieved in some samples [17] seems to suggest that the simple picture of $\mathrm{A}_{1} \mathrm{Mn}$ acceptor level according to Fig. 1 may not apply in this case.

Guided by the complex phenomenology very briefly discussed above and by available experimental data on III-V DMSs, several theoretical groups have performed detailed studies of these new ferromagnetic materials using both microscopic and effective Hamiltonian approaches. The results of some of this theoretical research are reviewed in the following paragraphs.

Ab-initio theories are an invaluable tool for studying the microscopic origins of ferromagnetism and for predicting electronic, magnetic, and structural groundstate properties of new compounds. The local density approximation (LDA) combined with disorder-averaging coherent-potential approximation (CPA) was used [18] to study GaAs and GaN DMSs doped with V, Cr, Mn, Fe, $\mathrm{Co}$, and Ni. A ferromagnetic ground-state was predicted for $\mathrm{V}, \mathrm{Cr}$, and $\mathrm{Mn}$ doping while $\mathrm{Fe}, \mathrm{Co}$, and Ni impurities 
were predicted to lead to a spin-glass state. The structural properties of $(\mathrm{Ga}, \mathrm{Mn})$ As have been calculated in the dilute limit and for hypothetical zinc-blende MnAs using pseudoatomic-orbital-based LDA algorithms [19]. The calculated lattice constant of MnAs is similar to the lattice constant of GaAs. This result appears to suggest that the relatively large compressive strains observed in $(\mathrm{Ga}, \mathrm{Mn})$ As epilayers grown on GaAs are caused by intrinsic defects rather than by the substitutional Mn.

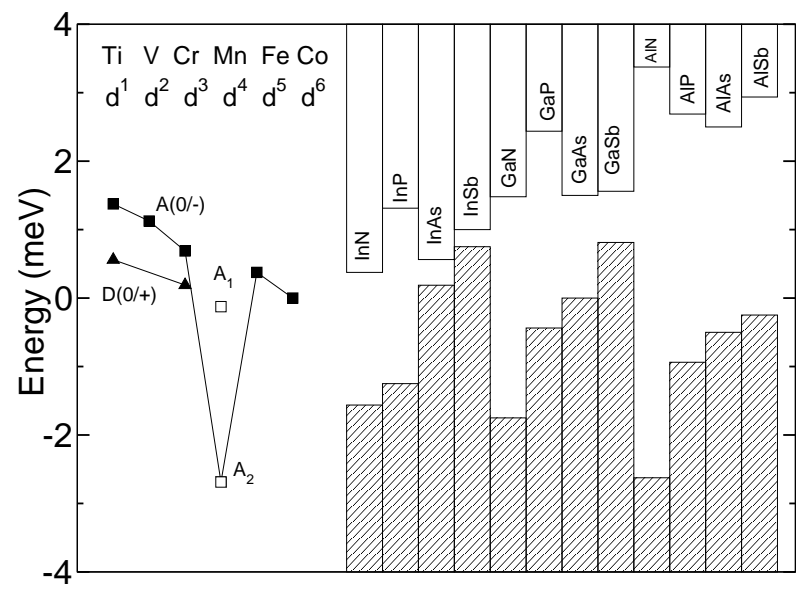

FIG. 1. Approximate positions of transition-metal donor $(\mathrm{D}(0 /+))$ and acceptor $(\mathrm{A}(0 /-))$ levels in III-V compounds [3]. Acceptor state labeled by $\mathrm{A}_{1}$ and the deep $\mathrm{A}_{2}$ level were obtained from spin-resonance experiments in GaP:Mn [4] and from photoemission in (Ga,Mn)As [5], respectively.

The electronic structure of (Ga,Mn)As supercells with $\mathrm{Mn}$ in the substitutional position, with As-antisites, or Mn-interstitial defects have been studied by various methods within the LDA $[19,21,22]$. Typical spinpolarized band and Mn 3d projected density-of-states results are shown in Fig. 2 [21] for substitutional Mn. It has been conclusively established by the LDA calculations that in the presence of substitutional Mn, the Fermi energy crosses the valence band near the $\Gamma$ point, introducing one hole per Mn. Clear theoretical evidence for As 4p-like band holes has been obtained in LDA+U calculations [20] and the Mn 3d density of states calculated by this method is in a good agreement with photoemission data [23]. As-antisite and Mn-interstitial defects act as a double donor, according to the microscopic calculations $[19,21,22]$, and, therefore, are the likely sources of carrier compensation observed in experiment. In summary, first-principles calculations support the picture of carrier induced ferromagnetism in some (III,Mn)V ferromagnets, but detailed predictions depend on the approximation used for exchange and correlations and also perhaps on technical details of the calculation. This activity is on-going and further progress should be expected.

Semi-phenomenological effective Hamiltonian theories in which the low-energy degrees of freedom are $\mathrm{Mn}$ ion $\mathrm{S}=5 / 2$ spins and valence band holes, discussed in the following paragraphs, have been developed to make quan- titative predictions for zero and finite temperature magnetic, transport and optical properties of bulk samples $[2,24]$ and heterostructures [25]. In (Ga,Mn)As, e.g., these models are partially justified by the negligible changes in the valence band structure of the host semiconductor observed in angle-resolved photoemission experiments [26]. The key term in the effective Hamiltonian description is a Kondo-like exchange interaction, $J_{p d} \sum_{I} \mathbf{S}_{I} \cdot \mathbf{s}\left(\mathbf{R}_{I}\right)$, which arises microscopically from sp-d hybridization and strong d-orbital Coulomb interactions. Here $\mathbf{S}_{I}$ is the $S=5 / 2$ local moment operator on $\mathrm{Mn}$ site $I$ and $\mathbf{s}(\mathbf{r})$ is the multi-band envelope function hole spin-operator $[27,28]$. The simplest version of this model combines mean-field theory with a virtual crystal approximation for the random distribution of Mn moments $[2,29,24,30]$. Illustrative results of the model are presented in Fig. 3 for the critical temperature in $(\mathrm{Ga}, \mathrm{Mn}) \mathrm{As}$ and $(\mathrm{In}, \mathrm{Mn})$ As. These calculations and the model predictions for the strain-engineered magnetic anisotropy, anomalous Hall conductivity [31], domain structure [32], and magnetic circular dichroism [27] are in good agreement with experiment.

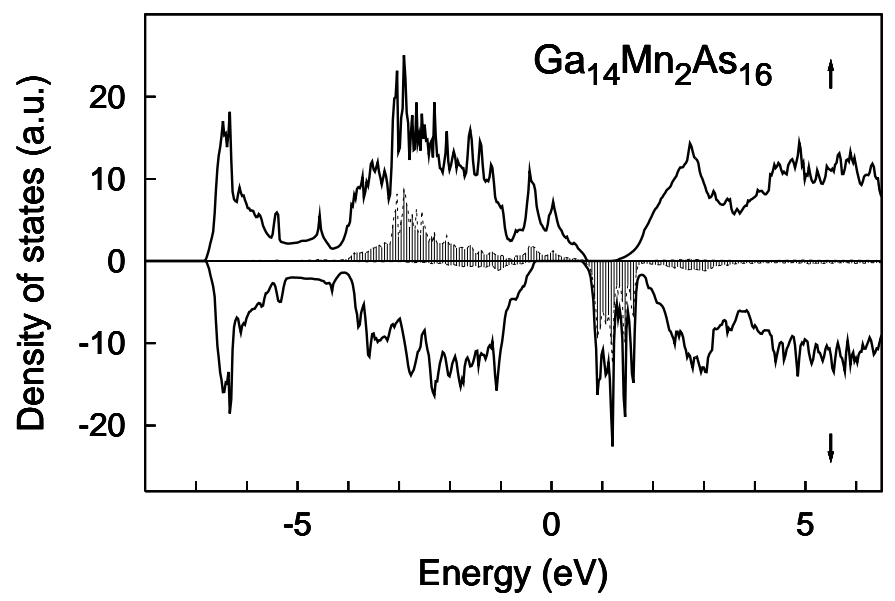

FIG. 2. LDA Spin-polarized density of states of $\mathrm{Ga}_{14} \mathrm{Mn}_{2} \mathrm{As}_{16}$ crystal with substitutional $\mathrm{Mn}$ atoms [21]. Grey areas represent the contribution from Mn 3d states. Energy is measured from the Fermi level.

The reliability of the standard mean-field approximation has been examined by accounting for the Coulomb interactions among holes [24], which enhance the critical temperature, and for correlations in Mn moment orientations which reduce the energetic cost of incompletely aligned spin configurations [33] and therefore lower the critical temperature. The calculations show that heavyhole - light-hole mixing is responsible for a relatively large spin stiffness, resulting in only $\sim 20 \%$ reduction of the critical temperature due to spin-wave fluctuations in arsenides and antimonides [34]. Moreover, the reduction is practically canceled by the enhancement of the critical temperature due to hole-hole interactions (see inset of Fig. 3) which explains the success of the mean-field 
theory in these ferromagnets [34].

Theoretical models beyond the virtual crystal approximation have been used to study the effects of disorder on transport properties, and magnetic properties in general, of the DMSs. The Boltzmann equation with Born approximation scattering rates have provided estimates of the anisotropic magnetoresistance effect (AMR) of order $\sim 1-10 \%$ [35], in good agreement with experiment $[11,13]$. AMR of a similar magnitude is predicted by Kubo formula for the ac-conductivity, as illustrated in Fig. 4. The key for understanding the transport and magnetic anisotropy effects is a strong spin-orbit coupling in the host semiconductor valence-band. The absolute dcconductivity estimates (see the left inset of Fig. 4) [35] are typically only 2-10 times larger than experimental values $[11,12,36]$. The discrepancy is likely partly due to inaccuracies in the model scattering amplitudes and multiple-scattering or localization effects omitted by this approximation. However experimental conductivities are still increasing as growth and annealing procedures are optimized so the discrepancy could be due mainly to unintended extrinsic disorder. Kubo formula calculations of infrared conductivities, illustrated in the right inset of Fig. 4, of metallic (Ga,Mn)As DMSs demonstrate that transverse f-sum rule measurements can be used to extract accurate values for the free carrier density [37]. The hole-fluid effective Hamiltonian theory discussed above uses no free parameters and is expected to be reliable in samples with high critical temperatures where the holes are metallic and their interaction with $\mathrm{Mn}$ and other impurities is effectively screened.

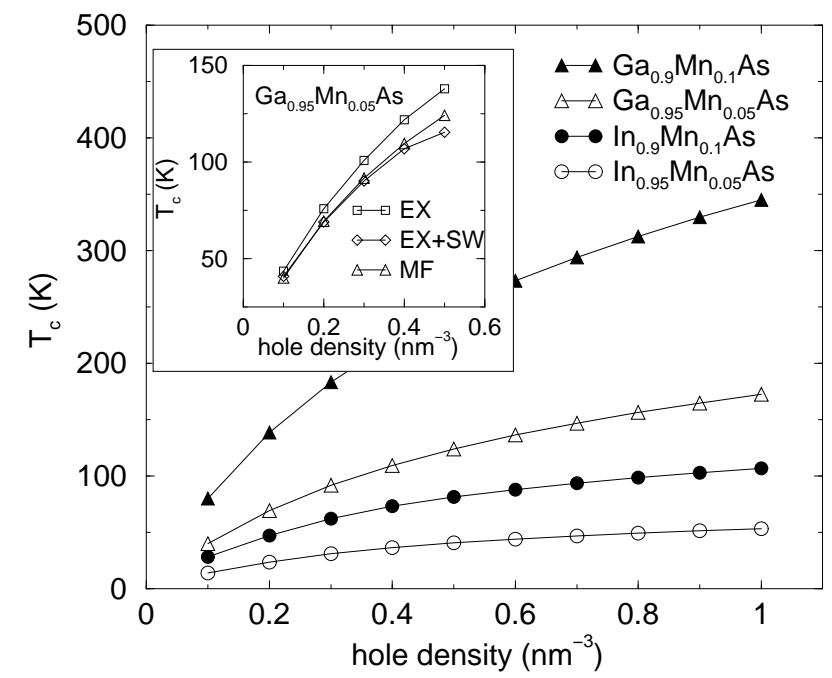

FIG. 3. Main plot: Mean-field critical temperature calculated using the effective Hamiltonian and virtual crystal approximations for (Ga,Mn)As (triangles) and (In,Mn)As (circles) DMSs as a function of the hole density [27,34]. Inset: Critical temperatures in ( $\mathrm{Ga}, \mathrm{Mn})$ As obtained by including hole-hole exchange interaction (EX) and spin-wave fluctuations $(\mathrm{EX}+\mathrm{SW})$ is compared to the mean-field results $(\mathrm{MF})$.

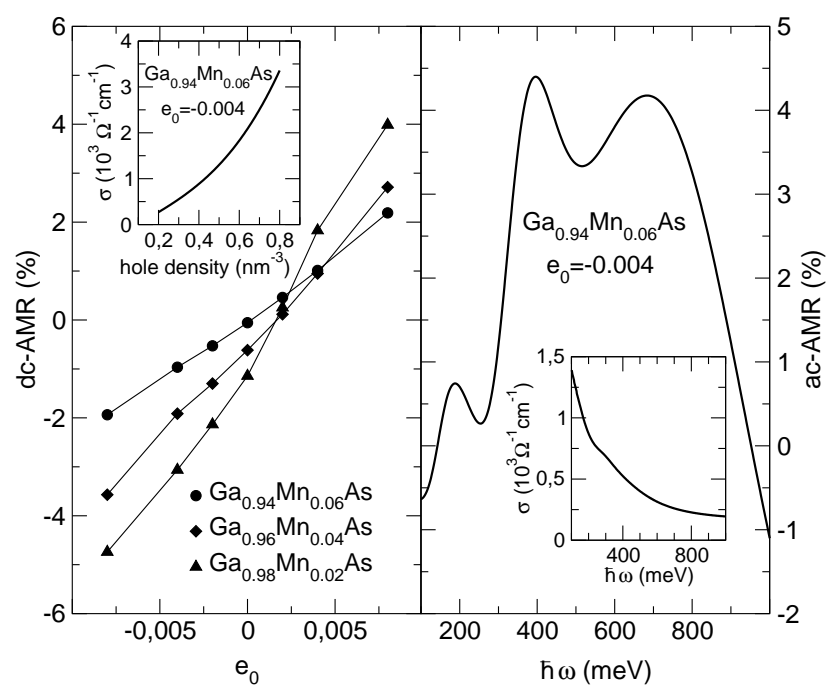

FIG. 4. Left panel: dc anisotropic magnetoresistance (AMR) as a function of strain for $50 \%$ compensated (due to As-antisites) (Ga,Mn)As DMSs. AMR is defined as the relative difference between resistivities for in-plane magnetization oriented along the current direction and for magnetization perpendicular to the plane of the DMS epilayer. $e_{0}$ is the relative difference between the substrate and the epilayer lattice constants. Inset shows the absolute conductivity for magnetization along the current direction. Right panel: ac-AMR as a function of frequency. Inset shows the absolute ac-conductivity.

More detailed studies of disorder effects in DMSs have combined Kondo description of the spin interactions with Monte Carlo techniques applied to both metallic [38] (high carrier density) and insulating [39] (low carrier density) regimes, and with the CPA [40] or spin-wave theory [41] for the metallic samples. The CPA calculations, which start from a tight-binding approximation band model and use RKKY theory of the effective MnMn interaction, predict a factor of 3 enhancement of the critical temperature due to disorder for typical $\mathrm{Mn}$ and hole densities in $(\mathrm{Ga}, \mathrm{Mn}) \mathrm{As}$ DMSs [40]. Other studies using dynamical mean field theory [42] suggest similar trends with some additional features when considering the effective $J_{p d}$ coupling as a variable. On the other hand, the random Mn distribution and spatial fluctuations of the magnetization suppress the ferromagnetic transition temperature in Monte Carlo simulations [38]. The non-collinear nature of the ground state due to disorder has been pointed out in the spin-wave theory calculations [41]. It was also suggested [43] that highly frustrated magnetic correlations may result from the strong spin-orbit interaction in the valence band and the spatial disorder.

The metal-insulator transition regime was explored by exact diagonalization techniques $[44,45]$. Consistent with experiment, the studies predict a metal-insulator transition for $1 \% \mathrm{Mn}$ doping in GaAs DMSs [44,45]. It 
has been emphasized [44] that defect correlations play an important role in the transition and the calculations $[44,45]$ have provided evidence in favor of the applicability of hole-fluid and impurity-band magnetic-polaron models on metallic and insulating sides of the transition respectively. Monte Carlo studies of the insulating phase have found spatially inhomogeneous magnetizations bellow the ferromagnetic critical temperature and an enhancement of the critical temperature due to disorder [39].

We acknowledge helpful discussions with Tomasz Dietl, and Jan Mašek who also provided figures presented in this review. The work was supported by the Grant Agency of the Czech Republic, DARPA, DoE, and the Welch Foundation.

[1] H. Ohno, Science 281, 951 (1998).

[2] T. Dietl, Semicond. Sci. Technol. 17, 377 (2002).

[3] J. Blinowski, P. Kacman, and T. Dietl, condmat/0201012.

[4] J. Kreissl, W. Ulrici, M. El-Metoui, A.-M. Vasson, A. Vasson, and A. Gavaix, Phys. Rev. B 54, 10508 (1996).

[5] J. Okabayashi, A. Kimura, O. Rader, T. Mizokawa, A. Fujimori, T. Hayashi and M. Tanaka, Phys. Rev. B 58, R4211 (1998).

[6] J.M. Langer, C. Delerue, M. Lannoo, and H. Heinrich, Phys. Rev. B 38, 7723 (1988).

[7] J. Szczytko, A. Twardowski M. Palczewska, R. Jablonski, J. Furdyna, H. Munekata, Phys. Rev. B 63, 085315 (2001).

[8] B. Beschoten, P.A. Crowell, I. Malajovich, and D.D. Awschalom, Phys. Rev. Lett. 83, 3073 (1999).

[9] H. Ohldag, V. Solinus, F.U. Hillebrecht, J.B. Goedkoop, M. Finazzi, F. Matsukura, and H. Ohno, Appl. Phys. Lett. 76, 2928 (2000).

[10] H. Ohno, J. Magn. Magn. Mater. 200, 110 (1999).

[11] D.V. Baxter, D. Ruzmetov, J. Scherschligt, Y. Sasaki, X. Liu, J. K. Furdyna, and C. H. Mielke. Phys. Rev. B 65, 212407 (2002).

[12] K.W. Edmonds, K.Y. Wang, R.P. Campion, A.C. Neumann, N.R.S. Farley, B.L. Gallagher, and C.T. Foxon, cond-mat/0209554.

[13] K.Y. Wang, K.W. Edmonds, R.P. Campion, L.X. Zhao, A.C. Neumann, C.T. Foxon, B.L. Gallagher, P.C. Main, and C.H. Marrows, to be published in Proc. 26th Int. Conf. on Physics of Semiconductors (Edinburgh, UK, 2002).

[14] H. Akai, Phys. Rev. Lett. 81, 3002 (1998).

[15] M.L. Reed, N.A. El-Masry, H.H. Stadelmaier, M.K. Ritums, M.J. Reed, C.A. Parker, J.C. Roberts, S.M. Bedair, Appl. Phys. Lett. 79, 3473 (2001).

[16] T. Sasaki, S. Sonoda, Y. Yamamoto, K. Suga, S. Shimiz, K. Kindo, H. Hori, J. Appl. Phys. 91, 7911 (2002).

[17] S. Sonoda, H. Hori, Y. Yamamoto, T. Sasaki, M. Sato,
S. Shimizu, K. Suga, and K. Kindo, cond-mat/0205560.

[18] K. Sato and H. Katayama-Yoshida, Semicond. Sci. Technol. 17, 367 (2002).

[19] S. Sanvito, G. Theurich, and N. Hill, Journal of Superconductivity 15, 85 (2002).

[20] J.H. Park, S.K. Kwon, and B.I. Min, Physica B 281/282, 703 (2002).

[21] F. Máca and J. Mašek, Phys. Rev. B 65, 235209 (2002).

[22] S.C. Erwin and A.G. Petukhov, cond-mat/0209329.

[23] J. Okabayashi, A. Kimura, T. Mizokawa, and A. Fujimori, T. Hayashi and M. Tanaka, Phys. Rev. B 59, R2486 (1999).

[24] J. König, J. Schliemann, T. Jungwirth, and A.H. MacDonald, in Electronic Structure and Magnetism of Complex Materials, eds. D.J. Singh and D.A. Papaconstantopoulos (Springer Verlag, Berlin 2002).

[25] Byounghak Lee, T. Jungwirth, and A.H. MacDonald, Semicond. Sci. Technol 17, 393 (2002).

[26] J. Okabayashi, A. Kimura, O. Rader, T. Mizokawa, A. Fujimori, T. Hayashi, and M. Tanaka, Phys. Rev. B 64, 125304 (2001).

[27] T. Dietl, H. Ohno, F. Matsukura, Phys. Rev. B 63, 195205 (2001).

[28] M. Abolfath, T. Jungwirth, J. Brum, and A.H. MacDonald, Phys. Rev. B 63, 054418 (2001).

[29] T. Dietl, H. Ohno, F. Matsukura, J. Cibert, and D. Ferrand, Science 287, 1019 (2000).

[30] T. Jungwirth, W.A. Atkinson, B.H. Lee, and A.H. MacDonald, Phys. Rev. B 59, 9818 (1999).

[31] T. Jungwirth, Q. Niu, and A.H. MacDonald, Phys. Rev. Lett. 88, 207208 (2002).

[32] T. Dietl, J. König, and A.H. MacDonald, Phys. Rev. B 64, R241201 (2001).

[33] J. König, H.H. Lin, and A.H. MacDonald, Phys. Rev. Lett. 84, 5628 (2000).

[34] T. Jungwirth, J. König, J. Sinova, J. Kučera, and A.H. MacDonald, Phys. Rev. B 66, 012402 (2002).

[35] T. Jungwirth, M. Abolfath, J. Sinova, J. Kučera, and A.H. MacDonald, cond-mat/0206416.

[36] S. J. Potashnik, K. C. Ku, R. Mahendiran, S. H. Chun, R. F. Wang, N. Samarth, and P. Schiffer, Phys. Rev. B 66, 012408 (2002).

[37] J. Sinova, T. Jungwirth, S.-R. Eric Yang, J. Kučera, and A.H. MacDonald, Phys. Rev. B 66, 041202 (2002).

[38] J. Schliemann, J. König, and A.H. MacDonald, Phys. Rev. B 64, 165201 (2001).

[39] R.N. Bhatt, M. Berciu, M.P. Kennett, and X. Wan, Journal of Superconductivity 15, 71 (2002).

[40] G. Bouzerar, J. Kudrnovský, and P. Bruno, condmat/0208596.

[41] J. Schliemann and A.H. MacDonald, Phys. Rev. Lett. 88, 137201 (2002); J. Schliemann, cond-mat/0209675.

[42] A. Chattopadhyay, S. Das Sarma, and A. J. Millis, Phys. Rev. Lett. 87, 227202 (2001).

[43] G. Zaránd and B. Jankó, Phys. Rev. Lett. 89, 047201 (2002).

[44] C. Timm, F. Schäfer, and F. von Oppen, Phys. Rev. Lett. 89, 137201 (2002).

[45] S.-R. Eric Yang, A.H. MacDonald, cond-mat/0202021. 